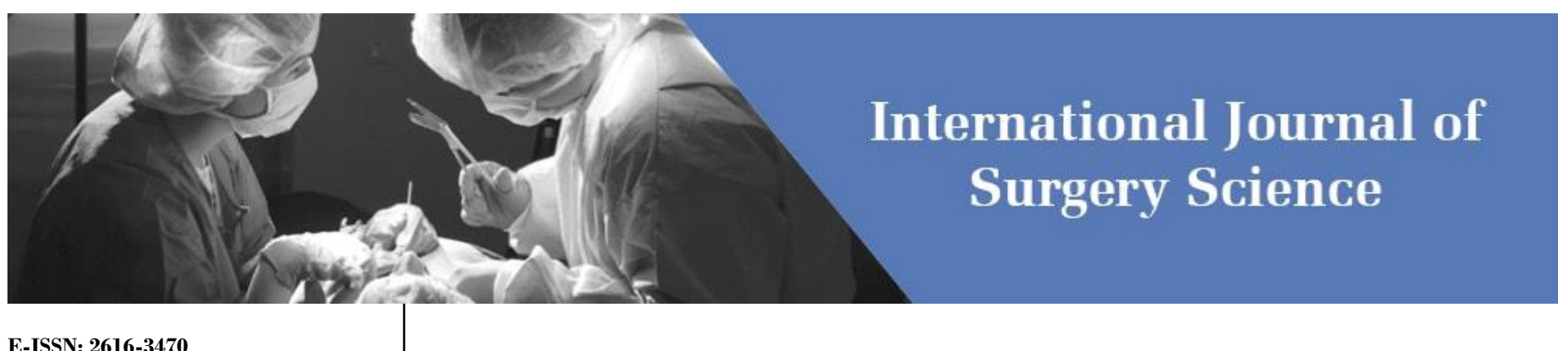

E-ISSN: 2616-3470

P-ISSN: 2616-3462

(C) Surgery Science

www.surgeryscience.com

2020; 4(2): 601-604

Received: 01-02-2020

Accepted: 05-03-2020

Dr. Ketan Vagholkar

Professor, Department of Surgery,

D.Y. Patil University School of

Medicine, Navi Mumbai,

Maharashtra, India

\section{Acute cholangitis: Diagnosis and management}

\section{Dr. Ketan Vagholkar}

DOI: https://doi.org/10.33545/surgery.2020.v4.i2g.447

\section{Abstract}

Acute cholangitis is a serious septic condition of the biliary tract. It is associated with obstruction of the biliary passages. Early diagnosis and assessment of the severity is essential. Aggressive supportive care, commencement of appropriate antibiotics, optimizing the function of various vital organ systems and early biliary drainage are pivotal in reducing the morbidity and mortality associated with this condition.

Keywords: Acute cholangitis diagnosis severity management

\section{Introduction}

Infections of the biliary tract are commonly encountered in surgical practice. Acute cholecystitis and acute cholangitis are the common biliary tract infections. The aetiology of acute cholangitis is variable ranging from stone disease to malignancy. Early diagnosis and prompt treatment is essential. The morbidity and mortality associated with acute cholangitis is quite high if diagnosis and treatment is delayed ${ }^{[1]}$. The aetiopathogenesis, diagnosis, severity assessment and management of acute cholangitis is discussed in the paper.

\section{Aetiopathogenesis}

Normal defence mechanisms in the biliary system preventing infection

There are a multiple mechanisms which protect the biliary system from infection ${ }^{[1,2,3]}$.

1. Continuous normal free flow of bile in the biliary passages keeps the intraductal pressure low and flushes out the passages thereby preventing bacterial concentration.

2. Bile salts have bacteriostatic activity.

3. Biliary epithelium secretes $\operatorname{Ig} \mathrm{A}$ which is protective and also secretes mucus which has antiadherent properties.

4. Kupffer cells in the biliary epithelium and tight junctions between the cholangiocytes prevent translocation of bacteria from the hepatobiliary system into the portal venous system.

5. The sphincter of Oddi prevents any migration of bacteria from the duodenum into the biliary system.

In an obstructed system there is a rise in the intraductal pressure due to stagnation of bile. The tight junctions between the cholangiocytes widen leading to entry by bacteria. The function of the Kupffer cells is also altered accompanied by fall in Ig A levels. This leads to successful bacterial invasion of the biliary passages ${ }^{[3,4]}$.

The normal choledochal pressure is 7 to $14 \mathrm{cms}$ of water. Complications develop when the pressure exceeds $25 \mathrm{cms}$ of water. There is cholangiovenous and cholangiolymphatic reflux. Bacteraemia followed by release of endotoxins occurs. There is release of inflammatory mediators like TNF, IL-1, IL-6, and IL-10 leading to hemodynamic insufficiency ${ }^{[4]}$.

The commonest cause of cholangitis is biliary obstruction. Choledocholithiasis, strictures and malignancy are the common causes of obstruction. However there is wide spectrum of causes for biliary obstruction. (Table 1)

Ascending cholangitis can occur due to migration of bacteria from the duodenum into the common bile duct ${ }^{[5]}$. Rarely do bacteria translocate from the portal vein into the bile duct. Other rare causes of cholangitis are primary biliary cholangitis, primary sclerosing cholangitis, Ig G4 related autoimmune cholangitis, and recurrent pyogenic cholangitis or oriental cholangiohepatitis ${ }^{[6]}$. 
Understanding the spectrum of bacterial flora causing cholangitis is essential for proper choice of antibiotic therapy. Coliforms are the predominant organisms isolated from bile in acute cholangitis. These include E. coli (25-50\%), klebsiella (15$20 \%)$, enterococcus species (10-20\%) and enterobacteriacae (5$10 \%)^{[6,7]}$.

Anaerobic organisms like bacteroides fragilis, Cl. Perfringens are found in elderly population or in those patients who have already undergone biliary tract surgery ${ }^{[8]}$. In a few cases, parasites have also been identified in bile. These include liver flukes clonorchis sinensis, opisthorchis viverrini, opisthorchis felineus and ascaris lumbricoides.

Recurrent acute cholangitis (oriental cholangiohepatitis) is an uncommon condition ${ }^{[9]}$. It is associated with pigment stones in the intrahepatic ducts predominantly in the lateral segment of the left lobe or in the posterior segment of the right lobe. Though the exact etiopathogenesis is unknown, it is associated with malnutrition and parasitic infestations such as ascaris lumbricoides and clonorchiasis. Transient portal bacteraemia allows entry of bacteria into the biliary system initiating a cycle of infection and stone formation. In $5 \%$ of cases there is a likelihood of development of cholangiocarcinoma ${ }^{[9]}$.

\section{Clinical features}

The common symptoms are right upper quadrant abdominal pain, high grade fever for more than 24 hours and jaundice. This is typically described as Charcot's triad. It has a sensitivity of $26.4 \%$ and specificity of $95.9 \%$ in diagnosing acute cholangitis $[9,10]$. When the severity of cholangitis escalates, hypotension and mental obtundation are seen in addition to pain, fever and jaundice. The five features together constitute Reynold's pentad. Charcot's triad is suggestive of acute cholangitis in 26.4 to $72 \%$ of cases ${ }^{[9,10]}$.

Physical examination will reveal high fever, tachycardia, hypotension, jaundice and altered sensorium in severe cases. Abdominal examination will reveal tenderness in the right hypochondriac and epigastric region.

\section{Investigations}

Complete blood count, liver function tests, PT/INR and renal profile are baseline haematological investigations. Inflammatory markers such as CRP and ESR are quite helpful [11, 12]. Blood cultures are useful in determining the spectrum of organisms causing cholangitis. Imaging modalities range from ultrasound imaging to MRCP ${ }^{[11,12]}$. The main aim of imaging is to identify the obstructing pathology. Collecting a bile sample during a drainage procedure is likely to yield a positive culture in 59$93 \%$ of acute cholangitis cases ${ }^{[13]}$.

\section{Diagnosis}

The new Tokyo guidelines (TG 13) help in ascertaining the diagnosis of acute cholangitis ${ }^{[9]}$. These take into consideration three parameters, inflammatory, cholestatic and imaging results $[9,10]$.

1. Systemic inflammation

A-1 Fever more than $38^{\circ} \mathrm{C}$ with shaking chills.

A-2 Laboratory data (Evidence of inflammatory response)

$\mathrm{WBC}<4000 / \mathrm{cmm}$ or $>10,000 / \mathrm{cmm}$.

$\mathrm{C}$ reactive protein $(\mathrm{CRP}) \geq 1 \mathrm{mg} / \mathrm{dl}$.

2. Cholestasis

B-1 Jaundice (Total bilirubin $\geq 2 \mathrm{mg} / \mathrm{dl}$ )

B-2 Laboratory data
Alkaline phosphatase $>1.5 x$ upper limit of normal (IU) $\gamma$ glutamyl transpeptidase $>1.5 x$ upper limit of normal(IU) AST/ALT > $1.5 \mathrm{x}$ upper limit of normal (IU)

3. Imaging

C-1 Biliary dilatation

C-2 evidence of aetiology in imaging (stone, stricture, stent)

Suspect diagnosis of acute cholangitis: one item in $\mathrm{A}+$ one item in $\mathrm{B}$ or $\mathrm{C}$.

Definitive diagnosis of acute cholangitis: one item in $\mathrm{A}+$ one in $\mathrm{B}+$ one in $\mathrm{C}$.

The sensitivity of TG 13 in diagnosis of acute cholangitis is $91.8 \%$ and specificity is $77.7 \%{ }^{[10]}$. The false positive rate for TG 13 is $5.9 \%, 11.9 \%$ for Charcot's triad ${ }^{[10]}$

\section{Assessment of severity}

Severity of cholangitis will determine the response to initial medical treatment and the possibilities of organ dysfunction. (TG13 guidelines) Hence it is very important to assess the severity of acute cholangitis ${ }^{[9,10]}$.

\section{Grade I (mild acute cholangitis)}

No organ dysfunction

Does not meet the criteria of moderate acute cholangitis

Responds to antibiotic treatment.

\section{Grade II (moderate acute cholangitis)}

No organ dysfunction

Does not respond to initial antibiotic treatment

Two out of the following five criteria should be present

1. $\mathrm{WBC}>12.000 / \mathrm{cmm}$ or $<4000 / \mathrm{cmm}$

2. High temperature $\geq 39^{\circ} \mathrm{C}$

3. Elderly (age $>75$ years)

4. Total bilirubin $\geq 5 \mathrm{mg} / \mathrm{dl}$

5. Hypoalbuminaemia $(<0.7 \times$ lower limit of normal $)$

\section{Grade III (severe acute cholangitis)}

Does not respond to initial medical treatment

Organ dysfunction present in at least one of the following organ systems.
1. CVS - dopamine infusion $\geq 5 \mu \mathrm{g} / \mathrm{kg} / \mathrm{min}$
2. CNS- altered consciousness
3. $\mathrm{RS}-\mathrm{PaO} 2 / \mathrm{FiO} 2$ ratio $<300$
4. Renal- oliguria / $\mathrm{S}$ creatinine $>2 \mathrm{mg} / \mathrm{dl}$
5. Hepatic: INR $>1.5$, platelet $<1$ lakh.

In acute suppurative cholangitis there is formation of pus in the biliary passages. On ERCP, pus is seen coming from the ampulla of Vater. Severe acute cholangitis or toxic cholangitis is seen in $5 \%$ of all cholangitis cases ${ }^{[11,12]}$

\section{Treatment}

The most important primary step towards treatment is aggressive resuscitation of the patient ${ }^{[13]}$. Majority of patients are hemodynamically compromised. Hence fluid resuscitation is essential. Supportive infusions of fresh frozen plasmas and vitamin $\mathrm{K}$ injections are helpful in preventing coagulopathy. Once haemodynamic stability is achieved then two issues need to be addressed, control of infection and relief of obstruction ${ }^{[13]}$. The choice of antibiotic for control of infection depends upon multiple factors ${ }^{[14]}$. These include
1. Renal status
2. Hepatic status
3. Drug allergies 


\section{Co morbidities}

5. Hospital acquired infections.

Initially empiric antibiotic therapy covering gram positive and anaerobic organisms needs to be started. There are a wide variety of antibiotics which can be used. These include piperacillin- tazobactam, ticaricillin- clavulanate, ceftriaxone with metronidazole and ampicillin with sulbactam. In patients sensitive to penicillin, ciprofloxacin with metronidazole, carbapenem or gentamicin with metronidazole can be used [14]. Once blood culture reports are available then specific antibiotic therapy can be commenced. Blood cultures are positive in 21$71 \%$ of acute cholangitis patients. Antibiotic therapy should be continued for ten days.

High biliary pressure caused by obstruction needs to be relieved at the earliest. Timing of drainage is dependent upon the grade of severity of cholangitis ${ }^{[15]}$.

Grade I needs drainage within 24 to 48 hours.

Grade II severity needs drainage at the earliest.

Grade III severity cases require optimization of organ systems followed by immediate drainage. This can be achieved by biliary drainage by a therapeutic ERCP. The various therapeutic options are

1. Endoscopic sphincterotomy with stone evacuation followed by stenting

2. Transpapillary biliary stenting for strictures

3. Replacement of blocked old stents with biofilm formation

4. Endoscopic nasobiliary drainage (ENBD)

5. Percutaneous transhepatic biliary drainage (PTBD)

6. Open surgical drainage ( $\mathrm{T}$ tube placement after laparotomy)

7. Rarely EUS guided drainage.

ENBD has a few advantages ${ }^{[15]}$.

A repeat cholangiogram can be done when the location of the stricture is unknown.

Purulent bile mixed with thick pus can be effectively drained.

The tube can be safely irrigated if clogged.

Bile aspirates for culture can be collected easily.

The main disadvantages are significant discomfort to the patient and chances that a confused patient will pull the tube out.

PTBD

PTBD is usually done after a failed ERCP. It can safely be done in patients with co morbidities especially in those patients who are unfit for ERCP ${ }^{[15]}$. The disadvantages of PTBD are patient discomfort, increase in hospital stay, and risk of biliary peritonitis, haemorrhage and infection.

PTBD is contraindicated in patients with ascites, coagulopathy and intrahepatic biliary obstruction.

Surgical drainage by way of choledochotomy with $\mathrm{T}$ tube placement is indicated when all options have failed ${ }^{[15,16]}$.

A balloon enteroscopic assisted ERCP with biliary drainage can be done in cases with obstructed Roux-en $\mathrm{Y}$ hepatico jejunostomy ${ }^{[16]}$.

EUS guided biliary drainage is considered when ERCP is unsuccessful as in cases of ampullary obstruction, gastric outlet obstruction and in a roux en Y hepaticojejunostomy ${ }^{[16]}$.

Oriental cholangiohepatitis poses both a diagnostic and therapeutic dilemma [17]. Administration of intravenous fluids and antibiotics is followed by intervention for biliary drainage. Stone extraction, stent placement, stricture dilatation and percutaneous biliary drainage after failed ERCP are various options ${ }^{[18]}$. Segmental hepatic resection for localized disease and liver transplantation for diffuse end stage liver disease can be done ${ }^{[17]}$. Prognosis depends upon the timing of biliary drainage, commencement of antibiotics and presence of co morbidities ${ }^{[19]}$. Poor prognostic factors are advanced age, high grade fever, leucocytosis, raised bilirubin levels and low albumin levels. Co morbidities associated with poor prognosis are cirrhosis, malignancy, liver abscesses, coagulopathy and raised creatinine levels. An IL 7 level less than 6 and a procalcitonin level greater than 0.5 are associated with increased mortality ${ }^{[20,21]}$. The mortality after drainage is $10 \%$ while after surgical intervention the mortality can rise to $30 \%{ }^{[16]}$.

Table 1: Aetiology of biliary obstruction

\begin{tabular}{|c|c|}
\hline Choledocholithiasis & $\begin{array}{c}\text { Benign or malignant } \\
\text { strictures of the bile ducts }\end{array}$ \\
\hline $\begin{array}{c}\text { Pancreatic cancer/ampullary } \\
\text { adenomas }\end{array}$ & Porta hepatis tumors/metastasis \\
\hline $\begin{array}{c}\text { Obstructed biliary stents (biofilm } \\
\text { formation, biliary sludge, } \\
\text { duodenal reflux) }\end{array}$ & Primary sclerosing cholangitis \\
\hline Mirizzi's syndrome & $\begin{array}{c}\text { Lemmel's syndrome (peri- } \\
\text { ampullary diverticulum causing } \\
\text { distal biliary obstruction) }\end{array}$ \\
\hline Round Worms (A lumbricoides) & Tape worm (T Saginata) \\
\hline AIDS cholangiopathy & $\begin{array}{c}\text { Strictured biliary-enteric } \\
\text { anastomosis }\end{array}$ \\
\hline Choledochocele & Post ERCP cholangitis \\
\hline
\end{tabular}

\section{Conclusion}

Acute cholangitis is a life threatening condition. Establishing the diagnosis and determining the severity is pivotal for formulating a therapeutic plan. Aggressive resuscitation, commencement of appropriate antibiotics and biliary drainage are the cornerstones of successful management.

\section{Conflict of interest: None \\ Funding: Nil}

Acknowledgements: I would like to thank Parth Vagholkar for his help in typesetting the manuscripts.

\section{References}

1. Mosler P. Management of acute cholangitis. Gastroenterol Hepatol (N Y). 2011; 7:121-123.

2. Kimura Y, Takada T, Strasberg SM, Pitt HA, Gouma DJ, Garden OJ et al. TG13 current terminology, etiology, and epidemiology of acute cholangitis and cholecystitis. J Hepatobiliary Pancreat Sci. 2013; 20:8-23.

3. Sung JY, Costerton JW, Shaffer EA. Defense system in the biliary tract against bacterial infection. Dig Dis Sci. 1992; 37:689-696.

4. Buyukasik K, Toros AB, Bektas H, Ari A, Deniz MM. Diagnostic and therapeutic value of ERCP in acute cholangitis. ISRN Gastroenterol. 2013; 2013:191729.

5. van den Hazel SJ, Speelman P, Tytgat GN, Dankert J, van Leeuwen DJ. Role of antibiotics in the treatment and prevention of acute and recurrent cholangitis. Clin Infect Dis 1994; 19:279-286.

6. Jain MK, Jain R. Acute bacterial cholangitis. Curr Treat Options Gastroenterol. 2006; 9:113-121.

7. Kinney TP. Management of ascending cholangitis. Gastrointest Endosc Clin N Am. 2007; 17:289-306.

8. Shi X, Vagholkar KR, Friess H, Uhl W, Buechler MW. Management of severe acute pancreatitis: Standards and Future Perspectives. Bombay Hospital Journal. 2001; 43:1627.

9. Kiriyama S, Takada T, Strasberg SM, Solomkin JS, Mayumi T, Pitt HA et al. Tokyo Guidelines Revision 
Committee. TG13 guidelines for diagnosis and severity grading of acute cholangitis (with videos). J Hepatobiliary Pancreat Sci. 2013; 20:24-34.

10. Kiriyama S, Takada T, Strasberg SM, Solomkin JS, Mayumi T, Pitt HA et al. Tokyo Guidelines Revision Committee. New diagnostic criteria and severity assessment of acute cholangitis in revised Tokyo Guidelines. J Hepatobiliary Pancreat Sci. 2012; 19:548-556.

11. Boey JH, Way LW. Acute cholangitis. Ann Surg. 1980; 191:264-270.

12. Lipsett PA, Pitt HA. Acute cholangitis. Front Biosci. 2003; 8:s1229-s1239.

13. Qureshi WA. Approach to the patient who has suspected acute bacterial cholangitis. Gastroenterol Clin North Am. 2006; 35:409-423.

14. Tanaka A, Takada T, Kawarada Y, Nimura Y, Yoshida M, Miura F et al. Antimicrobial therapy for acute cholangitis: Tokyo Guidelines. J Hepatobiliary Pancreat Surg. 2007; 14:59-67.

15. Nagino M, Takada T, Kawarada Y, Nimura Y, Yamashita $\mathrm{Y}$, Tsuyuguchi $\mathrm{T}$ et al. Methods and timing of biliary drainage for acute cholangitis: Tokyo Guidelines. J Hepatobiliary Pancreat Surg. 2007; 14:68-77.

16. Itoi $\mathrm{T}$, Tsuyuguchi $\mathrm{T}$, Takada $\mathrm{T}$, Strasberg SM, Pitt HA, Kim MH et al. Tokyo Guideline Revision Committee. TG13 indications and techniques for biliary drainage in acute cholangitis (with videos). J Hepatobiliary Pancreat Sci. 2013; 20:71-80.

17. Lim JH. Oriental cholangiohepatitis: pathologic, clinical, and radiologic features. AJR Am J Roentgenol. 1991; $157: 1-8$.

18. Zhang WZ, Chen YS, Wang JW, Chen XR. Early diagnosis and treatment of severe acute cholangitis. World $\mathrm{J}$ Gastroenterol. 2002; 8:150-152.

19. Lai EC, Tam PC, Paterson IA, Ng MM, Fan ST, Choi TK et al. Emergency surgery for severe acute cholangitis. The high-risk patients. Ann Surg. 1990; 211:55-59.

20. Tai DI, Shen FH, Liaw YF. Abnormal pre-drainage serum creatinine as a prognostic indicator in acute cholangitis. Hepatogastroenterology. 1992; 39:47-50.

21. Suwa Y, Matsuyama R, Goto K, Kadokura T, Sato M, Mori $\mathrm{R}$ et al. IL-7 and procalcitonin are useful biomarkers in the comprehensive evaluation of the severity of acute cholangitis. J Hepatobiliary Pancreat Sci. 2017; 24:81-88. 